\title{
Correlação entre a positividade do xenodiagnóstico artificial e a quantidade de sangue e triatomíneos utilizados no exame, em pacientes chagásicos crônicos
}

\author{
Correlation among the positivity of the artificial xenodiagnosis and the amount \\ of blood and triatomines used in the exam, in chronic chagasic patients
}

\author{
Yara Bandeira Azevedo Franco ${ }^{1}$, Ionizete Garcia da Silva ${ }^{1}$, Anis Rassi ${ }^{2}$, Alessandra Carla Rodrigues \\ Galvão Rocha', Heloisa Helena Garcia da Silva ${ }^{1}$ e Gustavo Gabriel Rassi ${ }^{3}$
}

\begin{abstract}
Resumo Estudou-se a correlação entre a positividade do xenodiagnóstico artificial e a quantidade utilizada de sangue e de triatomíneos, em 200 pacientes na fase crônica da doença de Chagas. Colheram-se 10 ou $40 \mathrm{ml}$ de sangue através de tubos a vácuo, heparinizados com 20,4UI, sendo realizado com 60 e 360 triatomíneos, respectivamente. Usou-se Dipetalogaster maximus, Triatoma infestans, Triatoma vitticeps e Rhodnius neglectus, nos estádios $1^{\circ}, 3^{\circ}, 3^{\circ}$ e $4^{\circ}$, respectivamente. A coproscopia dos triatomíneos foi realizada aos 30 e 60 dias após a aplicação. A positividade do xenodiagnóstico com o primeiro e o segundo métodos foi de $19 \%$ e $44 \%$, respectivamente, sendo altamente significativa a correlação entre a quantidade utilizada de sangue e triatomíneos e a positividade $(p<0,01)$. A xenopositividade nas faixas etárias variou de 9,7 a $100 \%$, sendo maior em jovens e adultos até 34 anos, e independente em relação ao sexo dos pacientes.
\end{abstract}

Palavras-chaves: Xenodiagnóstico artificial. Doença de Chagas. Fase crônica.

Abstract The aim of this study was to verify whether the amount of blood and number of triotomines used could improve the artificial xenodiagnosis performed in 200 chronic phase infected individuals. Ten or $40 \mathrm{ml}$ of peripheral blood was collected in heparinized (20.4 IU) vacuum tubes, and fed to 60 and 360 triatomines, respectively. Dipetalogaster maximus (1 $1^{\text {st }}$ instar, about 15 days after eclosion), as well as $3^{\text {rd }}$ instar Triatoma infestans and Triatoma vitticeps and the $4^{\text {th }}$ instar of Rhodnius neglectus were used. The faecal examinations were performed 30 and 60 days after xenodiagnosis procedure. The positivity with $10 \mathrm{ml}$ of blood was $19 \%$ and with $40 \mathrm{ml}, 44 \%$, from which it was concluded that a correlation existed between the amount of blood and the number of triatomines used $(p<0.01)$. The positivity of the xenodiagnosis ranged from 9.7 to $100 \%$, higher in young and adults up to 34 years old, but independent in relation to the sex of the chronic chagasic individuals studied.

Key-words: Artificial xenodiagnosis. Chagas' disease. Chronic phase.

Após ter sido demonstrada a viabilidade do uso do xenodiagnóstico artificial em rotina de laboratório 1516 para pacientes chagásicos crônicos, a sua utilização foi ampliada ${ }^{178}$. Em Goiás, onde a prevalência da doença de Chagas na fase crônica é alta, o xenodiagnóstico artificial faz parte da rotina diária de laboratório. A utilização desse método constitui avanço em relação ao método natural, com melhora da condição psicológica e maior adesão dos pacientes aos protocolos experimentais.
Mesmo existindo várias técnicas disponíveis para o diagnóstico da doença de Chagas, o xenodiagnóstico continua sendo útil, especialmente em ensaios clínicos, na seleção e acompanhamento de pacientes e verificação da eficácia de fármacos tripanosomicidas.

Este trabalho teve como objetivo analisar a possível correlação entre a positividade do xenodiagnóstico artificial, a quantidade de sangue e triatomíneos e a idade dos pacientes, visando esclarecer a sua sensibilidade, diminuir sua limitação de uso e orientar a sua aplicação clínica.

1. Laboratório de Biologia e Fisiologia de Insetos e Xenodiagnóstico do Instituto de Patologia Tropical e Saúde Pública (IPTSP) da Universidade Federal de Goiás (UFG); 2. Hospital São Salvador, Goiânia, GO, Hospital das Clínicas da Universidade Federal de Goiás; 3. Laboratório Atalaia, Goiânia, GO. Apoio financeiro: FUNAPE/UFG

Endereço para correspondência: Prof. Ionizete Garcia da Silva. Laboratório de Biologia e Fisiologia de Insetos e Xenodiagnóstico/IPTSP/UFG, Caixa Postal 131, 74001-970 Goiânia, GO

Fax: 5562 261-2675

e-mail:ionizete@iptsp.ufg.br

Recebido para publicação em 23/10/2001. 


\section{MATERIAL E MÉTODOS}

Triatomíneos. Utilizaram-se ninfas de Dipetalogaster maximus, Rhodnius neglectus, Triatoma infestans e $T$. vitticeps, criadas de acordo com a metodologia definida para cada espécie ${ }^{11} 171819$.

No xenodiagnóstico artificial, utilizando $10 \mathrm{ml}$ de sangue, foram aplicadas 60 ninfas de $D$. maximus, no $1^{\circ}$ estádio, 15 dias após a eclosão. No exame utilizando $40 \mathrm{ml}$ de sangue, usaram-se 90 ninfas de cada uma das espécies, sendo as de $D$. maximus, no $1^{\circ}$ estádio, as de $T$. infestans e de $T$. vitticeps no $3^{\circ} \mathrm{e}$ as de $R$. neglectus no $4^{\circ}$ estádio, totalizando 360 triatomíneos para cada xenodiagnóstico.

Pacientes e aspectos éticos. Foram avaliados 200 pacientes chagásicos crônicos não tratados especificamente, previamente diagnosticados por exame sorológico (IFI, HAI e ELISA), com acompanhamento médico no Hospital São Salvador e no Hospital das Clínicas da Universidade Federal de Goiás. Formaram-se dois grupos de 100 pacientes, que tiveram conhecimento prévio do estudo, e após anuência de cada paciente, colhiam-se $10 \mathrm{ml}$ de sangue no primeiro grupo, e $40 \mathrm{ml}$ no segundo. Os tubos com sangue foram codificados e enviados para exame laboratorial.
O sangue era coletado no antebraço, por punção na veia cubital, usando tubos a vácuo de $10 \mathrm{ml}$, heparinizados com $20,4 \mathrm{UI}$. No laboratório, o sangue era colocado no aparelho de $x e n o$ artificial $^{12}$ para alimentação das ninfas.

A coproscopia dos triatomíneos foi realizada aos 30 e 60 dias, utilizando-se o método das dejeções espontâneas ${ }^{14}$. Os insetos eram retirados dos frascos plásticos ${ }^{9}$ e transferidos para tubos de polietileno ${ }^{10}$ transparentes, que permitem melhor observação da alimentação. Colocavam-se quatro triatomíneos em cada frasco, devidamente identificados e numerados. Os insetos eram alimentados em galinha ${ }^{10}$ por um período de 20 minutos. Imediatamente após as dejeções, colhia-se uma amostra de fezes e urina de cada frasco, com um micropipetador de $50 \mu \mathrm{l}$, transferindo-a para uma microplaca de fundo plano e examinando-a ao microscópio de inversão (400x).

Análise estatística. Empregou-se o teste do quiquadrado, ao nível de significância de $5 \%$, para comparação da positividade do xenodiagnóstico artificial, com o sexo e as faixas etárias dos pacientes. Usou-se o teste entre duas proporções, ao nível de $1 \%$, para comparar a correlação da positividade entre o xeno realizado com 10 e $40 \mathrm{ml}$ de sangue.

\section{RESULTADOS}

A correlação da xenopositividade entre os dois grupos de chagásicos crônicos, utilizando os dois métodos de análise, foi altamente significativa, com $p<0,01$ (Tabela 1 e Figura 1).
Utilizando-se $10 \mathrm{ml}$ de sangue e 60 ninfas de D. maximus, a xenopositividade foi significativamente maior (42,9\%) nas faixas etárias de 24-34 anos e acima $(33,3 \%)$ de 68 anos (Tabela 2, Figura 1). A

Tabela 1 - Positividade do xenodiagnóstico artificial realizado por dois métodos, em 200 pacientes chagásicos crônicos, de acordo com o sexo.

\begin{tabular}{lccccc}
\hline & \multicolumn{2}{c}{$10 \mathrm{ml}$ de sangue e 60 ninfas } & & \multicolumn{2}{c}{$40 \mathrm{ml}$ de sangue e 360 ninfas } \\
\cline { 2 - 3 } \cline { 5 - 6 } Sexo & NP/NE & $\%$ & & NP/NE & $\%$ \\
\hline Masculino & $9 / 47$ & 19,1 & & $23 / 55$ & 41,8 \\
Feminino & $10 / 53$ & 18,9 & & $21 / 45$ & 46,7 \\
\hline Total & $19 / 100$ & 19,0 & & $44 / 100$ & 44,0 \\
\hline
\end{tabular}

NP- Número de pacientes positivos

NE- Número de pacientes examinados

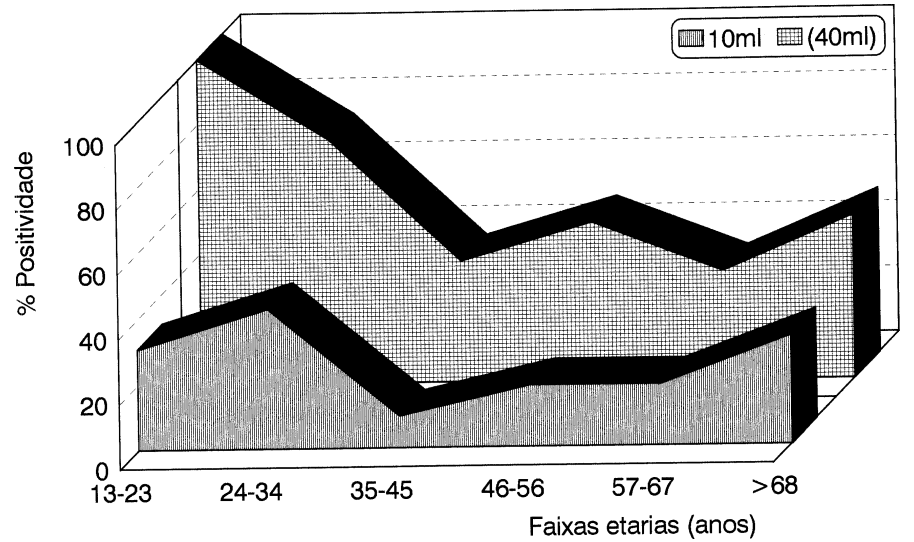

Figura 1 - Freqüência da positividade do xenodiagnóstico artificial em chagásicos crônicos, realizado com 10ml e 90 ninfas, e 40 $\mathrm{ml}$ de sangue 360 ninfas. 
Tabela 2 - Positividade do xenodiagnóstico artificial realizado com 10m/ de sangue e 60 ninfas, em 100 pacientes chagásicos crônicos, de acordo com a faixa etária e o sexo.

\begin{tabular}{|c|c|c|c|c|c|c|}
\hline \multirow[b]{2}{*}{ Faixa etária } & \multicolumn{2}{|c|}{ Masculino } & \multicolumn{2}{|c|}{ Feminino } & \multicolumn{2}{|c|}{ Total } \\
\hline & $\mathrm{NP} / \mathrm{NE}$ & $\%$ & NP/NE & $\%$ & $\mathrm{NP} / \mathrm{NE}$ & $\%$ \\
\hline $13-23$ & $1 / 7$ & 14,3 & $3 / 6$ & 50,0 & $4 / 13$ & 30,8 \\
\hline $24-34$ & $0 / 2$ & 0,0 & $3 / 5$ & 60,0 & $3 / 7$ & 42,9 \\
\hline $35-45$ & $3 / 18$ & 17,7 & $0 / 13$ & 0,0 & $3 / 31$ & 9,7 \\
\hline $46-56$ & $2 / 11$ & 18,2 & $1 / 13$ & 7,7 & $3 / 24$ & 18,8 \\
\hline $57-67$ & $2 / 7$ & 28,6 & $1 / 9$ & 11,1 & $3 / 16$ & 18,8 \\
\hline$>68$ & $1 / 2$ & 50,0 & $2 / 7$ & 28,6 & $3 / 9$ & 33,3 \\
\hline
\end{tabular}

NP- Número de pacientes positivos

NE- Número de pacientes examinados

xenopositividade do exame realizado com $40 \mathrm{ml}$ de sangue e 360 ninfas foi significativamente maior nas faixas etárias de 13-23 e 24-34 anos, com positividade de 100 e $75 \%$, respectivamente (Tabela 3, Figura 1).

A faixa etária de 35-45 anos apresentou a mais baixa positividade do xenodiagnóstico, em ambos os métodos (Figura 1). Na Tabela 2, observa-se que as faixas etárias de 35-45 e 57-67 tiveram xenopositividade estatisticamente iguais. Não houve diferença significativa na positividade do xenodiagnóstico entre pacientes chagásicos crônicos do sexo feminino e masculino (Tabela 1).

\begin{tabular}{|c|c|c|c|c|c|c|}
\hline \multirow[b]{2}{*}{ Faixa etária } & \multicolumn{2}{|c|}{ Masculino } & \multicolumn{2}{|c|}{ Feminino } & \multicolumn{2}{|c|}{ Total } \\
\hline & NP/NE & $\%$ & NP/NE & $\%$ & NP/NE & $\%$ \\
\hline $13-23$ & $1 / 1$ & 100,0 & $0 / 0$ & 0,0 & $1 / 1$ & 100,0 \\
\hline $24-34$ & $2 / 3$ & 66,7 & $1 / 1$ & 100,0 & $3 / 4$ & 75,0 \\
\hline $35-45$ & $9 / 23$ & 39,1 & $5 / 13$ & 38,0 & $13 / 38$ & 34,2 \\
\hline $46-56$ & $8 / 18$ & 44,4 & $9 / 17$ & 52,9 & $17 / 35$ & 48,6 \\
\hline $57-67$ & $2 / 9$ & 22,2 & $4 / 9$ & 44,4 & $6 / 18$ & 33,3 \\
\hline$>68$ & $1 / 1$ & 100,0 & $2 / 5$ & 40,0 & $3 / 6$ & 50,0 \\
\hline
\end{tabular}

NP- Número de pacientes positivos

NE- Número de pacientes examinados

\section{DISCUSSÃO}

Vários estudos foram realizados testando diferentes tipos de membranas ${ }^{24613}$ para a alimentação artificial de triatomíneos. Isso sinalizava a possibilidade de adaptação do método para realizar o xenodiagnóstico artificial. Apesar desses trabalhos apontarem a tripa de boi como a membrana mais favorável à alimentação artificial, a sua utilização na rotina foi prejudicada devido sua baixa disponibilidade e dificuldades de preservação. O preservativo masculino, não lubrificado e sem espermicida, demonstrou viabilidade de uso tanto para criação de triatomíneos ${ }^{12}$, quanto para a realização do xenodiagnóstico artificial ${ }^{1} 891516$ e testes com anticoagulantes ${ }^{5}$. Além disso, os resultados obtidos na rotina, durante vários anos no laboratório, aliados à sua disponibilidade no comércio, facilidade de conservação e praticidade no uso, foram itens decisivos na opção pelo seu emprego.

Os resultados obtidos apontam para fatos de interesse, como o aumento da sensibilidade do xenodiagnóstico artificial com a elevação do número de triatomíneos e da quantidade de sangue colhido. Quando as ninfas foram aumentadas em seis vezes e em quatro a quantidade de sangue, a positividade média do xenodiagnóstico elevou-se de 19 para $44 \%$. Nas faixas etárias de baixa positividade, de 35 a 45 anos, o aumento da sensibilidade foi maior, pois a xenopositividade elevou-se de $9,7 \%$ para $37,1 \%$, usando-se, respectivamente, 60 e 360 triatomíneos.

O xenodiagóstico realizado com $40 \mathrm{ml}$ de sangue e 360 ninfas recebeu a denominação rotineira de xenão. Este apresentou maior sensibilidade para detecção do Trypanosoma cruzi e, dependendo da faixa etária, a sensibilidade aumentou em até quatro vezes, como na faixa etária mais difícil de se detectar o tripanosoma, a de 35 a 45 anos, confirmando a hipótese de que, aumentando-se a quantidade de triatomíneos e de sangue, eleva-se a possibilidade de se encontrar o tripanosoma circulante.

Nos dois grupos de 100 pacientes, a idade foi um fator determinante na positividade do xenodiagnóstico (Figura 1). A maior xenopositividade ocorreu na faixa etária de 24-34 anos com o primeiro método, sendo também elevada, nessa faixa etária, com emprego do segundo método, cumprindo ressaltar, neste caso, resultou ainda maior na faixa de 13-23 anos. Esses 
resultados são concordantes com os da literatura ${ }^{348915}$, onde a faixa etária de 10-20 anos é a de maior positividade, decrescendo nas faixas etárias subseqüentes, e elevando-se nas faixas superiores a 50 anos. A freqüência da xenopositividade nas diversas faixas etárias pode ser atribuída ao tempo de infecção, à resposta imunológica associada à idade ou, ainda, à uma interação desses fatores.

A positividade do xenodiagnóstico artificial encontrada com as duas metodologias, foi independente em relação ao sexo dos pacientes, qualquer que fosse a faixa etária dos mesmos, observação concordante com outros trabalhos ${ }^{1520}$ e discordante dos resultados ${ }^{9}$ obtidos com 56 pacientes chagásicos, no qual a positividade do xenodiagnóstico foi maior no sexo masculino.

A xenopositividade média de $44 \%$ parece ser um fato comum, pois outros pesquisadores 3472021 encontraram índices similares. No entanto, deve ser ressaltado que $88 \%$ dos pacientes neste trabalho, encontravam-se nas faixas etárias de mais baixa parasitemia.

A correlação entre xenopositividade e a quantidade utilizada de triatomíneos e de sangue pode ampliar as perspectivas de uso do xenodiagnóstico artificial na seleção de pacientes para tratamento específico e na verificação de efeitos terapêuticos, hipótese que está sendo testada nos pacientes xenopositivos, com o benzonidazol (trabalho em avaliação).

Além disso, existem outras vantagens no uso do xenodiagnóstico artificial em relação ao natural: 1) tempo reduzido de ocupação do paciente, cerca de quatro minutos para coleta de sangue, versus 30 minutos na aplicação do natural; 2) possibilidade de uso de maior número de espécies e de espécimes de triatomíneos; 3) possibilidade de realização à distância; 4) ausência de risco inerente ao transporte de triatomíneos; 5) ausência de incômodo das picadas e de reações alérgicas; 6) possibilidade de utilização em qualquer idade, sendo mais adequado para crianças, geralmente mais inquietas e menos tolerantes a procedimentos demorados; 7) adequação para pacientes imunodeprimidos ou transplantados; 8) não exposição do paciente à curiosidade de circunstantes. Finalmente, quando é oferecida ao paciente a opção de escolha entre os métodos, essa recai, unanimemente, sobre o artificial.

\section{REFERÊNCIAS BIBLIOGRÁFICAS}

1. Azevedo YB, Rocha ACRG, Rassi A, Silva IG. Estudo comparativo entre a positividade do xenodiagnóstico artificial realizado com $10 \mathrm{ml}$ e $40 \mathrm{ml}$ de sangue. Revista da Sociedade Brasileira de Medicina Tropical 32(supl I): 326, 1999.

2. Campos R, Amato Neto V, Matsubara L, Moreira AAB, Pinto PLS. Estudos sobre o xenodiagnóstico "in vitro". I. Escolha de anticoagulantes e de membranas. Revista do Hospital das Clínicas da Faculdade de Medicina de São Paulo 43:101-103, 1988.

3. Castro CN, Alves MT, Macêdo VO. Importância da repetição do xenodiagnóstico para avaliação da parasitemia na fase crônica da doença de Chagas. Revista da Sociedade Brasileira de Medicina Tropical 16:98-103, 1983.

4. Cedillos RA, Torrealba JW, Tonn RJ, Mosca W, Ortegon A. El xenodiagnóstico artificial en la enfermedad de Chagas. Boletin de la Oficina Sanitaria Panamericana 93:240-249, 1982.

5. Isac E. Influência da heparina e do citrato de sódio no xenodiagnóstico artificial. Revista de Patologia Tropical 23:121-143, 1994.

6. Nussenzweig $\mathrm{V}$, Sontag R. Xenodiagnóstico artificial. Novo processo. Primeiros resultados positivos. Revista Paulista de Medicina 40:69-71, 1952.

7. Pineda JP, Luquetti A, Castro C. Comparação entre o xenodiagnóstico clássico e artificial na fase crônica da doença de Chagas. Revista da Sociedade Brasileira de Medicina Tropical 31:473-485, 1998.

8. Rocha ACRG, Azevedo YB, Silva APR, Rassi A, Silva IG. Aplicação do xenodiagnóstico artificial em chagásicos crônicos com a forma cardíaca, em estudo longitudinal. Revista da Sociedade Brasileira de Medicina Tropical 32(supl I):329, 1999.

9. Santos AH, Silva IG, Rassi A. Estudo comparativo entre o xenodiagnóstico natural e o artificial, em chagásicos crônicos. Revista da Sociedade Brasileira de Medicina Tropical 28:367373, 1995.
10. Silva IG. Influência da temperatura na biologia de triatomíneos. I. Triatoma rubrovaria (Blanchard, 1843) (Hemiptera, Reduviidae). Revista Goiana de Medicina 31:1-37, 1985.

11. Silva IG. Influência da temperatura na biologia de triatomíneos. XIII. Dipetalogaster maximus Uhler, 1894 (Hemiptera,Reduviidae). Anais da Sociedade Entomológica do Brasil 19:111-119, 1990.

12. Silva IG. Dispositivo para realização do xenodiagnóstico artificial. Revista de Patologia Tropical 20:35-38, 1991.

13. Silva IG, Luquetti AO, Silva HHG. Importância do método de obtenção das dejeções dos triatomíneos na avaliação da suscetibilidade triatomínica para Trypanosoma cruzi. Revista da Sociedade Brasileira de Medicina Tropical 26:19-24, 1993.

14. Silva IG, Rassi A, Azevedo YB, Galvão AC. Aperfeiçoamento do xenodiagnóstico artificial na avaliação da parasitemia: correlação existente entre a quantidade de sangue usada no xenodiagnóstico e a positividade. Revista da Sociedade Brasileira de Medicina Tropical 30:84-85, 1997.

15. Silva IG, Santos AH, Rassi A. Viabilidade do xenodiagnóstico artificial para exames de rotina em laboratório em substituição ao método tradicional. Revista da Sociedade Brasileira de Medicina Tropical 27:97, 1994.

16. Silva IG, Silva HHG. Influência da temperatura na biologia de triatomíneos. II. Rhodnius neglectus Lent, 1954 (Hemiptera, Reduviidae). Revista Goiana de Medicina 34:29-37, 1988.

17. Silva IG, Silva HHG. Influência da temperatura na biologia de triatomíneos. IV. Triatoma infestans (Klug, 1834) (Hemiptera, Reduviidae). Anais da Sociedade Entomológica do Brasil 17:443454, 1988b.

18. Silva IG, Silva HHG. Influência da temperatura na biologia de triatomíneos. X. Triatoma vitticeps Stal, 1859 (Hemiptera, Reduviidae). Revista Goiana de Medicina 34: 39-45, $1988 \mathrm{c}$. 
19. Silva IG, Silva HHG, Ostermayer AL, Rezende JM. Positividade do xenodiagnóstico de acordo com a faixa etária, sexo e forma clínica da doença de Chagas. Revista de Patologia Tropical 24:193-197, 1995.

20. Silva II. Sobre la conveniencia de realizar el xenodiagnostico fuera del organismo humano en todos los casos. Revista de la Facultad Medicina de Tucumán 1:405-415, 1958.
21. Souza HBWT, Moreira AAB, Matsubara L, Campos R, Amato Neto V, Pinto PLS, Takiguti CK. Estudo sobre o xeno "in vitro". II. Comparação do xeno "in vivo". Revista do Hospital das Clínicas da Faculdade de Medicina de São Paulo 43:165-167, 1988. 